\title{
Design-Optimization and Material Selection for a Proximal Radius Fracture-Fixation Implant
}

\author{
M. Grujicic, X. Xie, G. Arakere, A. Grujicic, D.W. Wagner, and A. Vallejo
}

(Submitted October 6, 2009; Accepted: 7 December 2009)

\begin{abstract}
The problem of optimal size, shape, and placement of a proximal radius-fracture fixation-plate is addressed computationally using a combined finite-element/design-optimization procedure. To expand the set of physiological loading conditions experienced by the implant during normal everyday activities of the patient, beyond those typically covered by the pre-clinical implant-evaluation testing procedures, the case of a wheel-chair push exertion is considered. Toward that end, a musculoskeletal multi-body inverse-dynamics analysis is carried out of a human propelling a wheelchair. The results obtained are used as input to a finiteelement structural analysis for evaluation of the maximum stress and fatigue life of the parametrically defined implant design. While optimizing the design of the radius-fracture fixation-plate, realistic functional requirements pertaining to the attainment of the required level of the devise safety factor and longevity/ lifecycle were considered. It is argued that the type of analyses employed in the present work should be: (a) used to complement the standard experimental pre-clinical implant-evaluation tests (the tests which normally include a limited number of daily-living physiological loading conditions and which rely on single pass/fail outcomes/decisions with respect to a set of lower-bound implant-performance criteria) and (b) integrated early in the implant design and material/manufacturing-route selection process.
\end{abstract}

Keywords musculoskeletal modeling and simulations, optimization, radius-fracture fixation-plate implant

\section{Introduction}

It is a common practice nowadays to judge the success of a surgery involving an implantable device (e.g., radius- or femoral-fracture fixation-plate implant, total hip replacement, etc.) by how quickly the patient can return to his/her normal activities of daily living following the surgical procedure (Ref 1). Another measure of the success of these surgeries is the longevity of the implanted device subjected to the physiological forces associated with the normal daily activities performed by the patient. However, as people are living longer and continuing to maintain active lifestyles, the paradigm of "everyday activities" must also evolve. This is critical since physiological loading conditions associated with these daily activities should be included in the pre-clinical implant-evaluation testing procedures. For example, long-term fatigue-controlled stability of hip implants has been evaluated under the conditions of normal walking (Ref 2-4), sit-to-stand (Ref 2), and stair climbing (Ref 3,4) and in a combination of these daily activities (Ref 5). However, only a relatively small number of the physiological loading conditions (derived from everyday

M. Grujicic, X. Xie, and G. Arakere, Department of Mechanical Engineering, Clemson University, Clemson, SC 29634-0921; A. Grujicic, Department of Bioengineering, Clemson University, Clemson, SC 29634-0905; and D.W. Wagner and A. Vallejo, Ozen Engineering, Inc., 1210 E. Arques Avenue, Suite: 207, Sunnyvale, CA 94085. Contact e-mail: mica.grujicic@ces.clemson.edu. activities of the patient), such as the ones just mentioned, are normally covered by the pre-clinical implant evaluation studies. The main reason for this is that the inclusion of more complex physiological loading conditions in the experimental preclinical implant evaluation testing procedures could be quite challenging and costly.

The additional shortcoming of the current pre-clinical implant evaluation studies is that they are used to mainly evaluate already designed and manufactured implant devices. This ensures that these devices will meet a predefined set of functional and longevity criteria. However, the results of these tests, unless the devise has failed to meet the performance/ longevity criteria, are rarely integrated in the overall implantdesign process. Consequently what is left unanswered is if the accepted new implant can meet the performance and longevity requirements under other physiological loading conditions associated with normal daily living and if the design (including the material selected for the implant) is optimal (with respect to its size, shape, placement, weight, cost, etc.).

The main objective of the present work is to demonstrate how musculoskeletal modeling can be used to generate physiological loading conditions not normally covered by the pre-clinical implant-evaluation tests, although they may be associated with the fairly normal daily activities. Within the present work, the recently developed novel technology for computer modeling of the human-body mechanics and dynamics, namely the AnyBody Modeling System (Ref 6) and its associated public domain library of body models are being fully utilized and further developed. In its most recent rendition (Ref 7), the AnyBody Modeling System enables creation of a detailed computer model for the human body (including all important components of the musculoskeletal system) as well as examination of the influence of different postures and the environment on the internal joint forces and muscle activity. 
The second main objective of the present article is to demonstrate how the loading conditions derived using musculoskeletal modeling can be utilized within a combined finite-element/design-optimization procedure to carry out optimization of the design of an implanted device. Specifically, optimal thickness, angular size, and placement of a proximal radius-fracture fixation-plate under wheel-chair push exertion loading conditions are investigated. Optimization of the implant thickness, angular size, and placement is carried out with respect to its ability to meet several functional requirements pertaining to both the necessary level of fractured-radius fixation and to meeting the longevity/lifespan constraints. Details regarding these functional requirements are presented in the next section.

The organization of the article is as follows. A brief overview of the AnyBody Modeling System is provided in Section 2.1. The musculoskeletal human-body model, the concepts of muscle recruitment and muscle activity envelope, the wheel-chair model and the issues related to human/wheelchair kinematics and contact interactions are discussed in Section 2.2-2.5. The definition of the musculoskeletal problem of a human propelling the wheel-chair analyzed in the present work is discussed in Section 2.5. The finite-element/designoptimization problem and analysis for the proximal radiusfracture fixation-plate implant are presented in Section 3. The results obtained in the present work are presented and discussed in Section 4. The main conclusions resulting from the present work are summarized in Section 5.

\section{Musculoskeletal Modeling and Simulations}

Within the present work, two distinct computational analyses are carried out. Within the first analysis (discussed in this section), a musculoskeletal investigation of a person propelling a wheel-chair is carried out. The resulting forces, moments, angular velocities, and angular accelerations, as functions of the time, acting on the fractured right radius of the person propelling the wheel-chair are next used in a finite-element/ design-optimization analysis of the proximal radius-fracture fixation-plate implant.

\subsection{The Anybody Modeling System (Ref 6)}

All the musculoskeletal modeling and simulation analyses carried out in the present work were done using the AnyBody Modeling System (Ref 6) developed at Aalborg University. The essential features of this computer program can be summarized as follows:

(a) The musculoskeletal model is typically constructed as a standard multi-body dynamics model consisting of rigid bodies, kinematic joints, kinematic drivers, and force/ moment actuators (i.e., muscles). The kinematic and dynamic behavior of this model can be determined using standard multi-body dynamics simulation methods;

(b) Complex geometries of the muscles and their spatial arrangement/interactions (e.g., muscles wrapping around other muscles, bones, ligaments, etc.) can be readily modeled within AnyBody Modeling System (Ref 6);

(c) It is well-established that a typical musculoskeletal system suffers from the so-called "muscle redundancy problem": i.e., the number of muscles available is generally larger than those needed to drive various body joints. Within the living humans and animals, this problem is handled by their Central Nervous System (CNS) which controls muscles activation/recruitment. To mimic this role of the CNS, the AnyBody Modeling System (Ref 6) offers the choice of several optimization-based muscle-recruitment algorithms;

(d) A typical musculoskeletal multi-body dynamics problem is solved using one of the computationally efficient inverse dynamics methods within which the desired body motion is prescribed while the muscle forces required to produce this motion is computed;

(e) Within the AnyBody Modeling System (Ref 6), the muscle recruitment problem is solved using an optimizationbased approach in the form:

Minimize the objective function:

$G\left(f^{(M)}\right)$

Subjected to the following constraints:

$C f=d$

$f_{i}^{(M)} \geq 0, \quad i \in\left\{1, \ldots, n^{(M)}\right\}$

where the objective function $G$ (a scalar function of the vector of $n^{(M)}$ unknown muscle forces, $\left.f^{(M)}\right)$, defines the minimization object of the selected muscle-recruitment criterion (assumed to mimic the one used by the CNS). Equation (2) defines the condition for dynamic mechanical equilibrium where $C$ is the coefficient matrix for the "unknown" forces/moments in the system while $d$ is a vector of the "known" (applied or inertia) forces. The forces appearing in vector $f$ in Eq (2) include the unknown muscle forces, $f^{(M)}$, and the joint-reaction forces, $f^{(R)}$. Equation (3) simply states that muscles can only pull (not push) and that the upper bound for the force in each muscle $f_{i}^{(M)}$ is the corresponding muscle strength, $N_{i}$.

(f) While there are a number of functional forms for the objective function, $G$, the one used in the present work is the so-called " $\min / \max$ " form within which the objective function (to be minimized) is defined as the maximum muscle activity defined for each muscle $i$ as $f_{i}^{(M)} / N_{i}$, i.e.:

$G\left(f^{(M)}\right)=\max \left(f_{i}^{(M)} / N_{i}\right)$

This formulation offers several numerical advantages over other popular forms of $G$ and, in addition, it appears to be physiologically sound. That is, under the assumption that muscle fatigue is directly proportional to its activity, Eq (1) and (4) essentially state that muscle recruitment is based on a minimum muscle-fatigue criterion. Also, this expression for $G$, has been found to asymptotically approach other formulations of $G$ [e.g., the so-called "Polynomial" form (Ref 8)].

(g) The problem defined by Eq (1)-(4) can be linearized using the so-called "bound formulation" (Ref 9) resulting in a linear programming problem with muscle forces and joint reaction forces as free variables. Relations between these two types of forces are next used to 
eliminate the joint reaction forces yielding a linear programming problem with the number of unknowns equal to the number of muscles in the system; and

(h) While for a fairly detailed full-body model containing around one thousand muscles, this constitutes a medium-to-large size problem which can be readily solved by a variety of design-optimization methods (e.g., Simplex, Interior-point methods, etc.), the $\mathrm{min} /$ max problem is inherently indeterminate and must be solved iteratively. This can be rationalized as follows: The min/max criterion only deals with the maximally activated muscles and with muscles which help support the maximally activated muscles. Since the system, in general, may contain muscles that have no influence on the maximum muscle activity in the system, the forces in these muscles are left undetermined by the $\min / \mathrm{max}$ formulation presented above. To overcome this shortcoming, the muscle-recruitment optimization problem is solved iteratively, so that each iteration eliminates the muscles with uniquely determined forces and the procedure is repeated until all muscle forces are determined.

\subsection{Musculoskeletal Human-Body Model}

The human body musculoskeletal model used in the present work was downloaded from the public domain AnyScript Model Repository (Ref 10). The model was originally constructed by AnyBody Technology using the AnyBody Modeling System (Ref 6) following the procedure described in details by Damsgaard et al. (Ref 7$)$.

2.2.1 Model Taxonomy. The musculoskeletal humanbody model includes: (a) an arm/shoulder assembly containing 114 muscle units on each side of the body and having a morphology defined by Van der Helm (Ref 11), (b) a spine model developed by de Zee et al. (Ref 12) comprising sacrum, all lumbar vertebrae, a rigid thoracic-spine section, and a total of 158 muscles, and (c) a pelvis and lower extremity model with a total of 70 muscles. In total, the model contains more than 500 individual muscle units and, hence, can be considered as a fairly detailed description of the human musculoskeletal system. The anthropometrical dimensions of the model are selected in such a way that they roughly correspond to a 50th percentile European male.

2.2.2 Segments and Joints. Within the model, the bodies (referred to as the "segments" within the AnyBody Modeling System) are treated as rigid with their mass/inertia properties derived from mass and shape of the associated bone and the soft tissue that is allotted to the bone. Joints in the human body are treated as idealized frictionless kinematic constraints between the adjoining segments. Both standard kinematic joints (e.g., spherical joints for the hips, hinge joints for the knees, etc.) as well as specially developed joints (e.g., those used to represent kinematic constraints associated with floating of the scapula on the thorax) are employed.

2.2.3 Muscles. Muscles are treated as string contractile force-activation elements which span the distance between the origin and the insertion points through either the via points or by wrapping over the surfaces which stand on their way. Muscle wrapping problem is treated using a shortest-path contact-mechanics algorithm. Due to the fact that the problem considered in the present work is dynamic, muscles are modeled as being non-isometric (i.e., muscle strength is considered to be a function of the body posture and the rate of contraction). Also, passive elasticity of muscles (i.e., the resistance of the muscles to stretching) was considered.

2.2.4 Model Validations. The mechanics of the model is implemented as a full three-dimensional Cartesian formulation and includes inertial and gravity body forces. Integral validation of whole-body musculoskeletal models is very difficult to conduct. To the best knowledge of the present authors, validation of the whole-body musculoskeletal model is still lacking (due to major challenges which would be associated with such validation). However, various subsystems of the whole-body model were validated separately. For example: (a) The lumbar spine model was validated by de Zee et al. (Ref 12) by comparing the model prediction with in vivo L4-5 intradiscal pressure measurements of Wilke et al. (Ref 13); (b) de Jong et al. (Ref 14) validated the lower extremity model by comparing model-predicted muscle activations and pedal forces with their experimental counterparts obtained in pedaling experiments; and (c) The shoulder model was validated in the early work of Van der Helm (Ref 11).

\subsection{The Muscle Activity Envelope}

As originally recognized by An et al. (Ref 15), the $\min / \max$ muscle-recruitment formulation, discussed in Section 2.1, defines effectively a minimum fatigue criterion as the basis for muscle recruitment, i.e., the aim of the proposed musclerecruitment strategy is to postpone fatigue of the "hardestworking" muscle(s) as far as possible. The physiological consequence of this strategy is that muscles tend to form groups with muscles within the same group having comparable activity levels. In particular, in the muscle group associated with the maximum muscle activity there will be usually many muscles which, in a coordinated manner, carry a portion of the load comparable with their individual strengths. Consequently, in this group, many muscles will have the same activity level, which will be referred to as "the muscle activity envelope". The linearity of the reformulated $\mathrm{min} / \mathrm{max}$ criterion discussed earlier guarantees that the optimization problem defined by Eqs (1)-(3), is convex and, hence, that the solution to the problem is unique and corresponds to the global optimum. In other words, there is no other muscle recruitment strategy which can reduce the muscle-activity envelope further. Moreover, since the muscle activity envelope represents the maximum muscle activation in the model, it can be interpreted as the fraction of maximum voluntary contraction necessary to support the imposed loads (wheel-chair push-exertion and gravity forces, in the present case) while maintaining the prescribed posture.

\subsection{Wheel-Chair Model}

The wheel-chair is modeled using four segments, i.e., the two wheels, the seat, and the back-rest. These segments are constrained to the global reference frame and used merely for the visual representation of the wheel-chair. That is, these segments were not directly used in the musculoskeletal analysis of the wheel-chair propulsion problem. Instead, as explained in the next section, the interactions between the human and wheel-chair was modeled implicitly by prescribing the timedependent motion to various human-body segments and the time-dependent force experienced by the right hand during wheel-chair propulsion. 


\subsection{Musculoskeletal Definition of the Wheel-Chair Propulsion Problem}

As mentioned above, wheel-chair/human-body interactions were modeled implicitly by prescribing time-dependent motions and forces to the body, Fig. 1. Specifically, seven points in the body (three of which were located on the thorax and one on the right humerus, the right radius, the right ulna, and the right hand each) were used in the present case. Timedependent positions of these points were obtained in a set of motion-capture laboratory experiments. Within these experiments, a male individual experienced with the use of a wheelchair was instrumented with seven reflective markers on the outside of his spine, the right shoulder and the right arm. After a wheel-chair specialist adjusted the wheel-chair to match the anthropometry of the person propelling the chair, the individual was asked to propel the wheel-chair at a comfortable speed while seven cameras recorded motion of the upper-body markers. Meanwhile, motion capture measurements were taken in order to locate and track the position of the reflective markers. The markers-position data recorded as a function of time were then used as input to the AnyBody Modeling System to drive the human body model during the simulated wheelchair ride, Fig. 2 (Ref 16). The remainder of the body was then allowed to acquire the appropriate posture by adjusting kinematics of the spine in accordance with the so-called "spinal rhythm" algorithm. Within this algorithm, a single input, the pelvis-thorax angle, is used to determine the three rotational-joint angles of adjacent vertebrae (under a condition that the passive-elastic elements of the spine are able to force the spine to act cinematically as an elastic beam). The physical soundness of the spinal-rhythm algorithm for the seating

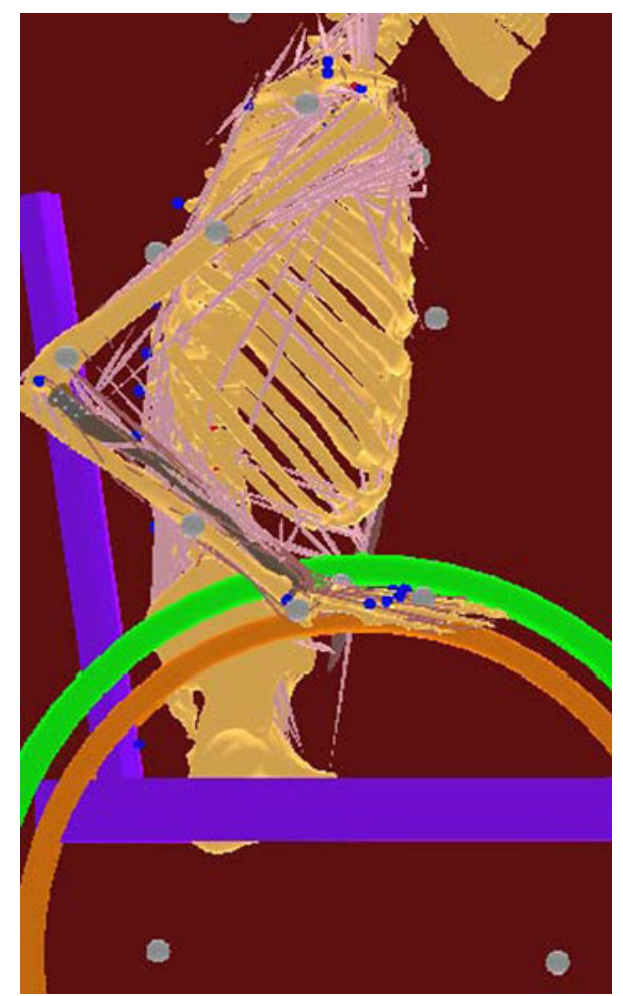

Fig. 1 Scaled musculoskeletal model of a person propelling the wheel-chair (not scaled to size) posture has been validated by Rasmussen and de Zee using motion capture experiments (Ref 17).

Time-dependent loads experienced by the right hand during wheel-chair propulsion were obtained using laboratory experiments and involved force-sensing push-rims operated by the laboratory participant. The data were collected at a sampling frequency of $240 \mathrm{~Hz}$. The collected data were next filtered using a forth-order, $20 \mathrm{~Hz}$ low-pass Butterworth filter and then the resulting forces in Newtons were obtained via simple conversion from the collected data in Volts.

The markers-position and hand-force recorded data were finally fitted using an eight-order B-spline function, which enabled determination of instantaneous values of the marker locations and hand-push forces as required in the musculoskeletal analysis of wheel-chair propulsion.

\section{Finite-Element and Design-Optimization Procedures}

As mentioned earlier, the results of the musculoskeletal wheel-chair propulsion analysis in the form of: (a) fracturedradius/implant assembly reference-frame/center-of-mass coordinates, orientations, velocities and accelerations as a function of time; (b) temporal variations of the spatial coordinates of the muscle attachment/via points and of the muscle forces; and (c) temporal variations of the spatial coordinates of the radius/elbow and radius/wrist joint reaction forces and

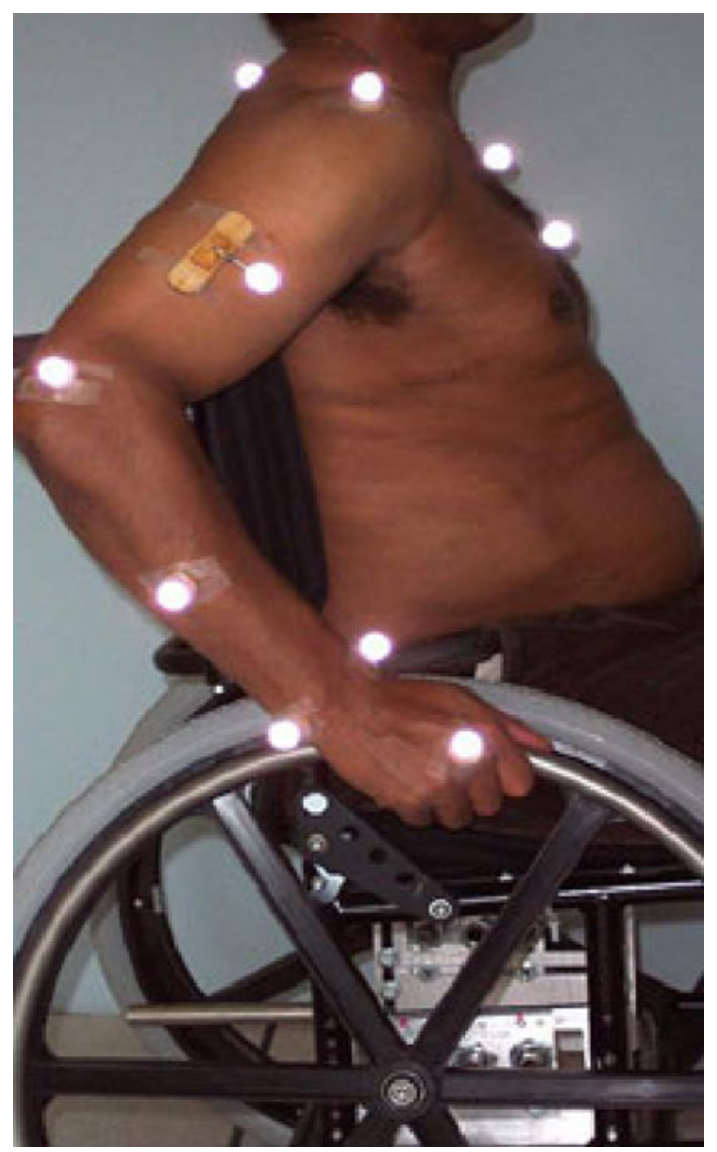

Fig. 2 Participant in the wheel-chair motion-capture experiments (Ref 16) 
moments, are exported from the AnyBody Modeling System and used, as input, in a finite-element/design-optimization analysis of the proximal radius-fracture fixation-plate implant. Some details pertaining to the finite-element/design-optimization analysis are presented in the remainder of this section. In Fig. 3, the names and the spatial locations of the muscleattachment/via points and the elbow and wrist joints are provided for the right radius. In this figure, there are 45 muscle attachment/via points and two joint points. Moments are transferred to the radius only at the two joint points since muscles, being contractile linear elements, can each provide only a force.

In Fig. 4, a close-up is provided of the right radius along with the adjoining bones. As can be seen, the radius contains a proximal (i.e., next to the elbow) fracture and it is fixed with a lateral fracture fixation-plate implant. The implant is attached to the two segments of the fractured radius using six screws.

\subsection{Finite Element Model and Analysis}

3.1.1 The Model. The finite element model analyzed consisted of a fractured right radius, a fixation-plate implant, and six locking screws. Typical finite element meshes used are displayed in Fig. 5. The radius, the plate, and each of the screws were discretized using ca. 13,000 ten-node second-order tetrahedral solid elements, ca. 2,200 twenty-node second-order brick elements, and ca. 200 twenty-node second-order brick elements, respectively.

To apply the muscle forces and joint-reaction forces and moments to the radius, each muscle-attachment/joint point is combined with a neighboring section of the radius surface to form a coupling. In this way, forces/moments acting at a muscle-attachment/joint point are transferred to the radius over a larger surface area preventing (unrealistic) stress-concentration artifacts.

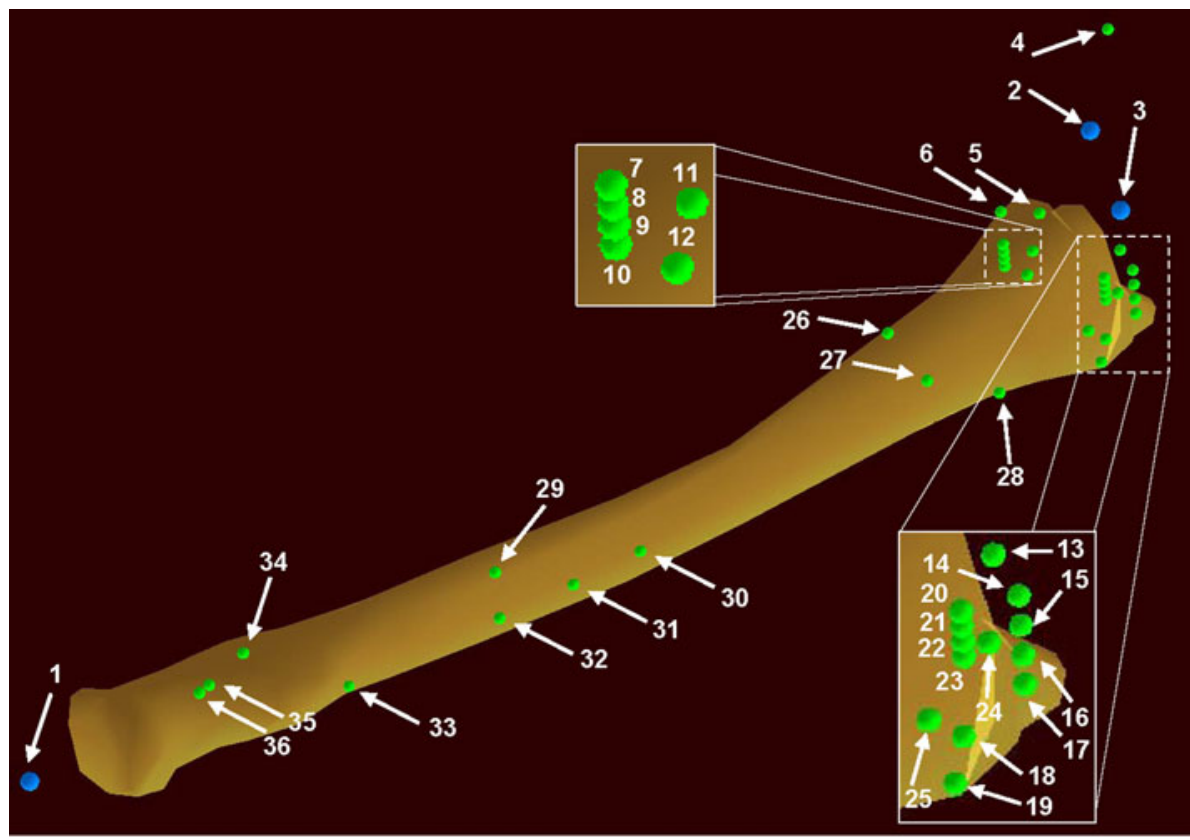

- PointPS2 Node (Elbow Joint Node)

2 - PS Node (Distal Pronation Supination Joint Node)

3 - Wrist Joint Node

4 - MedialExtensorCyl Node

5 - Via_Extensor_Indicis Node

6 - Via_Extensor_Digiti_Minimi Node

7- Via_Extensor_Digitorum Digit5 Node

8 - Via_Extensor_Digitorum_Digit4

9 - Via_Extensor_Digitorum_Digit3

10 - Via_Extensor_Digitorum_Digit2 Node

11-Via Extensor Pollicis Longus

Node/Nia Abductor_Pollicis_Longus

NodeNia_Extensor_Carpi_Radialis_Longus Node

12 - Via_Extensor_Carpi_Radialis_Brevis Node

13-Via_Palmaris_Longus Node

14 - Via_Flexor_Digitorum_Superficialis_Digit5 Node

15 - Via_Flexor_Digitorum_Superficialis_Digit4 Node

16 - Via_Flexor_Digitorum_Superficialis_Digit3 Node

17-Via_Flexor_Digitorum_Superficialis_Digit2 Node

18 - Via_Flexor_Pollicis_Longus Node

19-I_Brach_rad_1 Nodell_Brach_rad_2 Node
20 - Via_Flexor_Digitorum_Profundus_Digit5 Node 21- Via_Flexor_Digitorum_Profundus_Digit4 Node 22 - Via_Flexor_Digitorum_Profundus_Digit3 Node 23 - Via_Flexor_Digitorum_Profundus_Digit2 Node 24-Via_Flexor_Carpi_Radialis Node

25- I_Pron_quadr_2 Node

26 - O_Extensor_Pollicis_Brevis Node

27 - SupinatorCyl Node

28 -I_Pron_quadr_1 Node

29 - BicepsCyl Node

30 - O_Flexor_Pollicis_Longus Node

31 - I_Pronator_teres_caput_humeral_1 Node/

I_Pronator_teres_caput_humeral_2

NodeA_Pronator teres_caput ulnare_1 Node

32 -1_Supinator_ulna_part_2

Nodell_Supinator_humerus_part_2 Node

$33-1$ Supinator ulna part_1

Nodefl_Supinator_humerus_part_1 Node

34 - I_Biceps_LH Node/l Biceps_SH Node

35 - RadiusMuscleCy Node

36 - PronatorTeresCyl Node

Fig. 3 Spatial location of various muscle attachment points to the right radius 


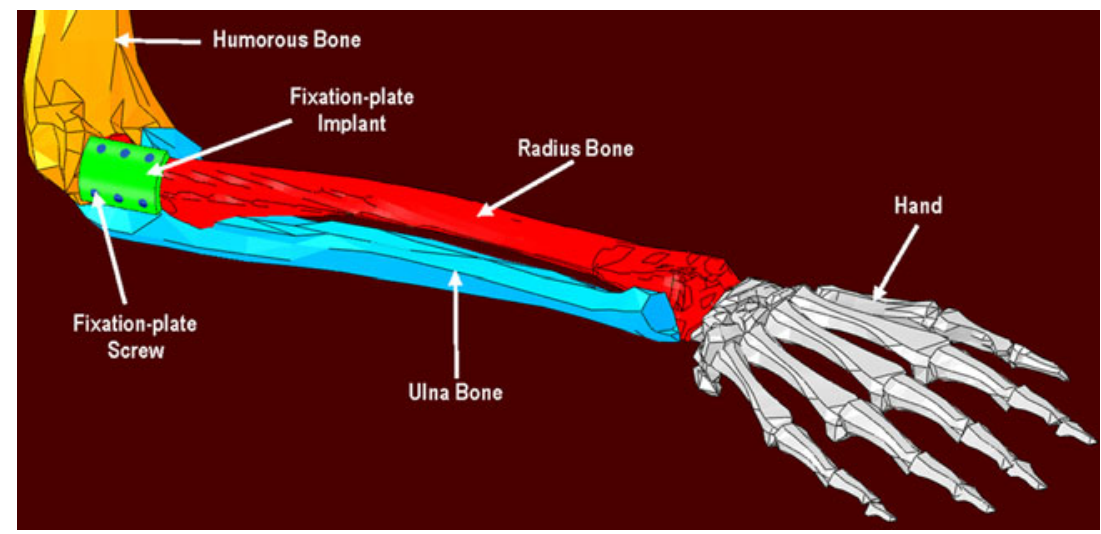

Fig. 4 A close-up view of the fractured right radius (with a lateral fixation-plate implant and locking screws) and the adjoining bones at one time instant during a wheel-chair propulsion-simulation run

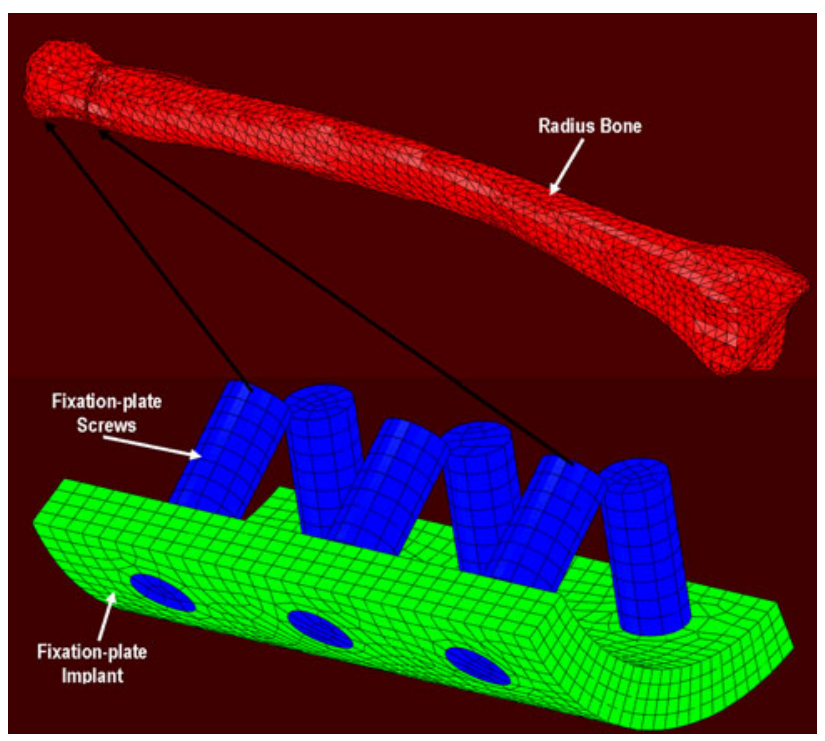

Fig. 5 Typical finite-element meshes for the radius, the fixationplate implant and six screws used in the quasi-static analysis of the implant longevity

To fasten the screws to the fixation plate and to the two bone segments, the outer surfaces of the screws are tied to the mating surfaces of the plate/radius. In other words, a "perfect fastening" condition is assumed to have been achieved using the screws.

To prevent sections of the bottom surface of the fixationplate implant between the screws from penetrating the radius, a "penalty-type" contact algorithm was employed. Within this algorithm, penetration of the contacting surfaces is opposed by a set of contact springs. Any level of contact pressure can be transmitted through the contact interface. Shear stresses are transmitted across the contact-interface in accordance with the Coulomb friction law.

3.1.2 Material Models. The fixation-plate implant and the seven screws are assumed to be made of Ti-6Al-4V, a Ti-based alloy which is commonly used in fractured-bone fixation applications. Ti-6Al-4V is modeled as a linear-elastic/ ideal-plastic material.

To provide a higher level of realism to the analysis, it is recognized that radius is built of two types of bone tissues

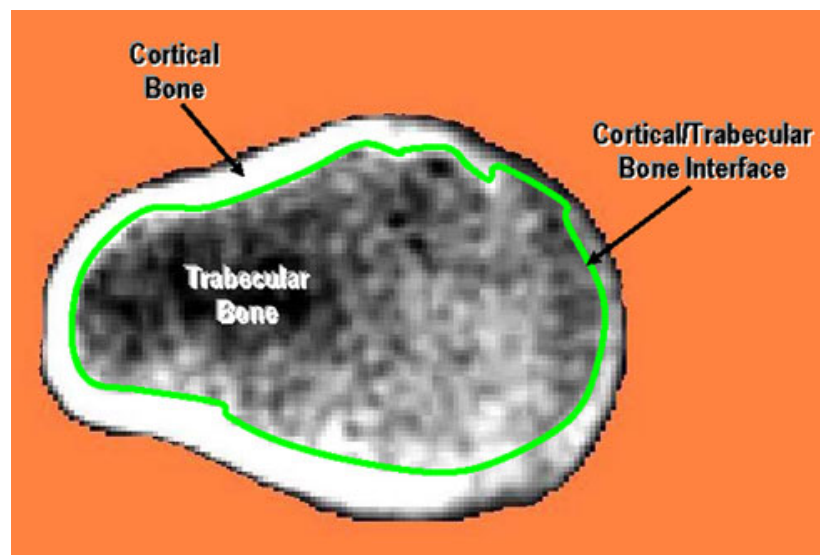

Fig. 6 A typical Computed Tomography (c7) scan of the radius showing the presence of two bone tissues (the cortical and the trabecular) and the associated density variation within each bone tissue

(cortical and trabecular) and that density (and hence mechanical properties) of the two types of bone tissues are spatially non-uniform. To obtain the necessary data for defining the cortical-bone/trabecular-bone dividing surfaces and the spatial distribution of the density within each of the two bone tissues, Computed Tomography (CT) scans of the radius bone were analyzed (Ref 18). An example of the CT scan of the radius bone is shown in Fig. 6. For each bone tissue, the local grayscale level is proportional to the local density. CT images like the one displayed in Fig. 6 are analyzed using the Medical Imaging Software Mimics (Ref 19). Within Mimics, the two bone tissues are differentiated by assigning two nonoverlapping gray-scale ranges, one for each bone tissue. Then, the gray-scale of each pixel within the two bone tissues is quantified using the Hounsfield Unit (HU) value. The latter are next converted into the corresponding bone-density values as: $\rho=1.9 \mathrm{HU} / 1,700$, where the bone-density $\rho$ is given in $\mathrm{g} / \mathrm{cm}^{3}$. Lastly, the relations listed in Table 1 in (Ref 18) were used to compute the Young's modulus as a function of the local density within the two bone tissues. A constant value of $v=0.3$ was used for both bone tissues. No plasticity within the radius was considered. In other words, it was assumed that the radius was made of two isotropic heterogeneous linear-elastic materials. 
Table 1 Functional relations used to compute, from CT-scans, density and the Young's modulus in the cortical and trabecular bones of the radius

\begin{tabular}{llc}
\hline $\begin{array}{l}\text { Bone } \\
\text { region }\end{array}$ & $\begin{array}{c}\text { Density ( } \boldsymbol{\rho}) \text { vs. } \\
\text { Hounsfield unit } \\
(\mathbf{H U}) \text { relationship }\end{array}$ & $\begin{array}{c}\text { Young's modulus }(\boldsymbol{E}) \\
\text { vs. density }(\boldsymbol{\rho}) \text { relationship }\end{array}$ \\
\hline $\begin{array}{c}\text { Cortical } \\
\text { bone }\end{array}$ & $\rho\left(\mathrm{g} / \mathrm{cm}^{3}\right)=1.9 \frac{\mathrm{HU}}{1700}$ & $E(\mathrm{MPa})=-13,430+$ \\
$\begin{array}{c}\text { Trabecular } \\
\text { bone }\end{array}$ & $\rho\left(\mathrm{g} / \mathrm{cm}^{3}\right)=1.9 \frac{\mathrm{HU}}{1700}$ & $E(\mathrm{MPa})=1310\left(\rho\left(\mathrm{g} / \mathrm{cm}^{3}\right)\right)^{1.40}$ \\
\hline
\end{tabular}

3.1.3 The Analysis. The results of the AnyBody-based multi-body dynamic wheel-chair propulsion analysis over a single hand-push cycle are exported at 100 equal time intervals. For each of these intervals, a quasi-static finite-element analysis of the radius/fixation-plate/screws assembly is carried out. At each of these time steps, the following AnyBody output information was used as input boundary/loading conditions (a) Spatial position of the radius/fixation-plate/screw assembly and the associated muscle-attachment and joint-reaction points;

(b) muscle forces and joint-reaction forces and moments; and (c) the radius/plate/screws assembly (linear and angular) velocities and accelerations.

The aforementioned AnyBody output data were used within the finite element model as follows: (a) the spatial position data were used to correctly position the finite-element model and the points for the application of concentrated forces and moments; (b) The muscle-force and joint-reaction force/moment data were used to define concentrated-load type of boundary conditions; and (c) The velocity and acceleration data were used to define distributed (gravity, inertia, and centripetal) loading conditions.

The finite-element analysis results were used to determine: (a) if the fixation-plate implant has suffered (unacceptable) plastic deformation; (b) if the two contacting fractured surfaces of the radius have intruded into each other (also an unacceptable scenario); and (c) the stress-state of the most critical elements (elements which control the fatigue life of the fixation-plate implant).

All the calculations pertaining to the quasi-static response of the radius/plate/screws assembly are done using ABAQUS/ Standard, a commercially available general-purpose finiteelement program (Ref 20).

\subsection{Design-Optimization Analysis}

One of the main objectives of the present work was to carry out optimization of the proximal radius-fracture fixation-plate implant design. The overall implant-design/geometry was parameterized in terms of the following three parameters: (a) plate thickness (2-4 mm); (b) angular plate-size $\left(35-45^{\circ}\right)$; and (c) angular plate-position $\left(10-170^{\circ}\right)$, Fig. 7: The information listed within the parentheses denotes the range of the parameter in question with the $100^{\circ}$ angular plate-position being located on the proximal lateral side of the radius. Since the plate was modeled as a solid structure, changes in its geometry entailed continued re-meshing of this component during optimization. In addition, since the screws length also changed during optimization (in order to comply with the changing plate thickness), the screws had to be re-meshed as well. Likewise, since the depth/location of the holes within the radius changed during implant optimization, the two bone fragments had to be repeatedly re-meshed.

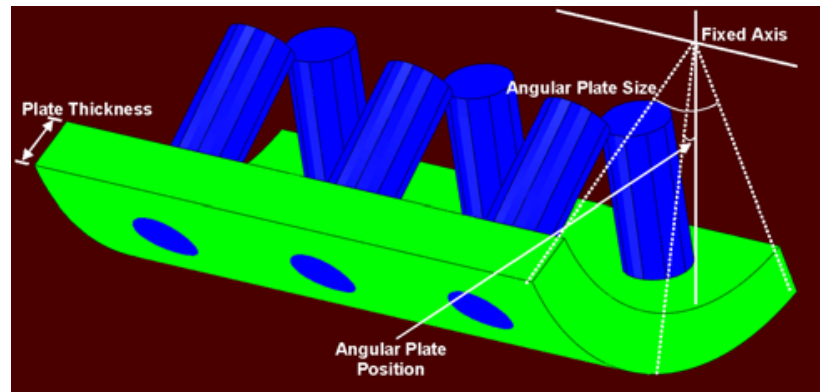

Fig. 7 Definitions of the fractured-radius fixation-plate design/positioning variables used in the present implant optimization work

3.2.1 Structural Optimization. Structural optimization is a class of engineering optimization problems in which the evaluation of an objective function(s) or constraints requires the use of structural analyses (typically a finite element analysis, $F E A$ ). In compact form, the optimization problem can be symbolically defined as (Ref 21$)$ :

Minimize the objective function $f(x)$

Subjected to the non-equality constraints $g(x)<0$ and

to the equality constraints $h(x)=0$

with the design variables $x$ belonging to the domain $D$

where, in general, $g(x)$ and $h(x)$ are vector functions. The design variables $x$ form a vector of parameters describing the geometry of a part/component. For example, $x, f(x), g(x)$, and $h(x)$ can be part dimensions, part weight, a stress condition defining the onset of plastic yielding, and constraints on part dimensions, respectively. Depending on the nature of design variable in question, its domain $D$ can be continuous, discrete or a mixture of the two. Furthermore, a structural optimization may have multiple objectives, in which case the objective function becomes a vector function.

Structural optimizations can be classified in many different ways. One of these classifications distinguishes between topology, size, and shape optimization methods.

Topology Optimization: Topology optimization which is typically applied at the conceptual stage of part design represents the design domain as the continuum mixture of a solid material and "voids" and the optimal design is defined with respect to the distributions of the mixture density within the design space (e.g. Ref 22).

Size Optimization: Within size optimization approach, the dimensions that describe part geometry are used as design variables, $x$. The application of size optimization is, consequently, mostly used at the detailed part-design stage where only fine tuning of the part geometry is necessary. Size optimization is typically quite straightforward and it generally requires no re-meshing of the finite element models during optimization iterations.

Shape Optimization: Shape optimization which is also mostly used at the detailed part-design stage, allows the changes in the boundary of part geometry. The boundaries are typically represented as smooth parametric curves/surfaces, since irregular boundaries typically deteriorate the accuracy of finite element analysis or may even cause the numerical instability of optimization algorithms. Because the product geometry can change dramatically during the optimization process, the automatic re-meshing of finite element models is 
usually required. Structural shape optimization methods are generally classified as: (a) direct geometry manipulation and (b) indirect geometry manipulation approaches. In the direct geometry manipulation approaches, design variable $x$ is a vector of parameters representing the geometry of part boundary, e.g., the control points of the boundary surfaces. In the indirect geometry manipulation approaches, design variable $x$ is a vector of parameters that indirectly defines the boundary of the product geometry. A comprehensive review of shape optimization based on the direct and the indirect geometry manipulation approaches can be found in Ref 23 .

3.2.2 Fractured-Radius Fixation-Plate Design/Positioning Optimization. The fractured-radius fixation-plate design/ positioning optimization problem was defined as follows: The plate mass is to be minimized while ensuring that during wheelchair propulsion no plastic deformation in the plate takes place, no interpenetration of the two fractured radius segments occurs and that no high-cycle fatigue failure will take place after a preselected number of hand-push crank revolutions (set to two million cycles, in accordance with a simple analysis presented in Section 4).

The fractured-radius fixation-plate design/positioning optimization is carried out as a two-step procedure:

(a) within the first step, a full-factorial Design of Experiments (DOE) study was conducted using three design variables: the plate thickness $(2.0,2.5,3.0,3.5$, and $4.0 \mathrm{~mm})$, plate angular size $(35,37,39,41,43$, and 45 degrees), and plate angular position (10, 55, 100, 145, and 190 degrees), where the numbers within the parentheses represent the levels of each of the three design variables. The full-factorial matrix includes $5 \times 6 \times 5=$ 150 discrete designs. Three of these designs, each associated with the same level of plate thickness $(3 \mathrm{~mm})$ and the angular size $\left(45^{\circ}\right)$ but at three different angular positions $\left(10^{\circ}, 100^{\circ}\right.$, and $\left.190^{\circ}\right)$ are displayed in Fig. 8; and

(b) once the DOE results are obtained and fitted to a higherorder mathematical function (the so-called "Response Surface"), the resulting meta-model is subjected to the standard three design variable optimization procedure. In other words, the optimization algorithm is applied to the higher-order mathematical function without the use of costly finite-element analyses. An example of the resulting maximum von Mises response surface for the complete ranges of the fixation-plate design at three different angular positions is shown, respectively, in Fig. 8(a)-(c).

The fixation-plate design/positioning optimization problem was implemented into and solved using HyperStudy (Ref 24), a general purpose multi-disciplinary multi-objective optimization software. For a defined optimization problem, this software invokes a pre-selected DOE algorithm (a fullfactorial method, in the present case) and an optimization algorithm [the Adaptive Response Surface Method (Ref 25), in the present case], prepares the appropriate input files for the finite-element analysis, launches the finite-element solver and reads and interprets the finite-element results in order to construct the response surface in the DOE case and to determine the immediate search direction in the designoptimization case.

3.2.3 Implant Fatigue-Life Prediction. As mentioned earlier, one of the fractured-radius fixation-plate designoptimization constraints pertains to the attainment of a
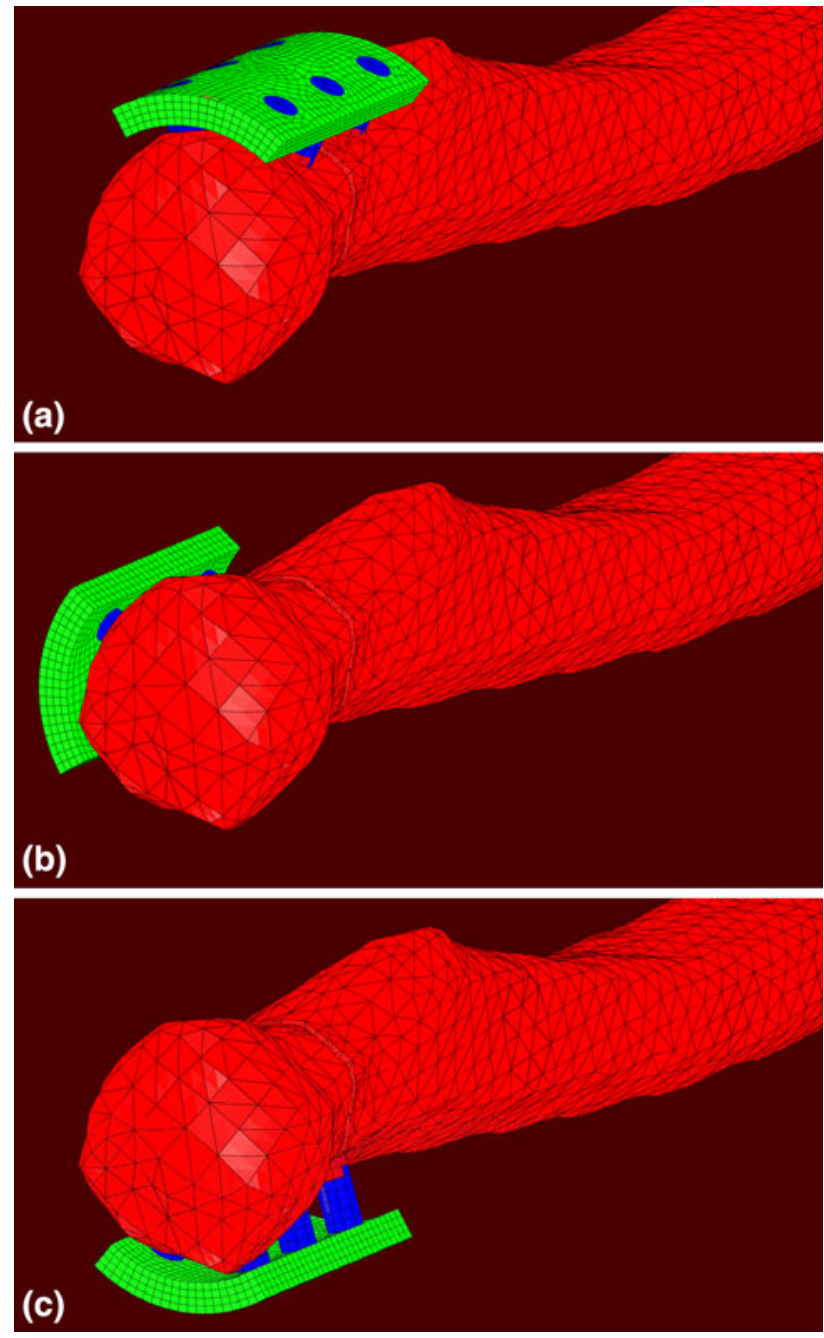

Fig. 8 Examples of the fixation-plate design at three different angular positions. Please see text for details

pre-selected lifecycle. Since this lifecycle is expected to be high-cycle fatigue controlled, a fatigue-based lifecycle prediction procedure had to be developed in the present work. The first step in this direction was to examine the temporal behavior of the muscle forces and reaction forces and moments during a single hand-push cycle. An example of the results obtained for the two (elbow and wrist) joint-reaction force and moment components is displayed in Fig. 9(a) and (b). Simple examination of the results displayed in these figures show that the temporal evolution of various forces and moments is not in phase and that these forces/moments are not associated with constant amplitude. These findings have important consequences to the type of fatigue-life prediction analysis which should be employed. Firstly, the non-constant nature of the load amplitude implies that a cycle-counting procedure [e.g., the so-called Rainflow Cycle-counting Analysis (Ref 26)] should be employed in order to represent (highly irregular) time-dependent loading as a collection of constant-amplitude (fixed mean-value) loading cycles. Secondly, since temporal evolution of the various muscle forces and joint-reaction forces and moments are out of phase, not only the magnitude of stresses/strains at an arbitrary point in the radius/plate/screw assembly varies as a function of time, but also the orientation of the associated principal coordinate system is time variant. 


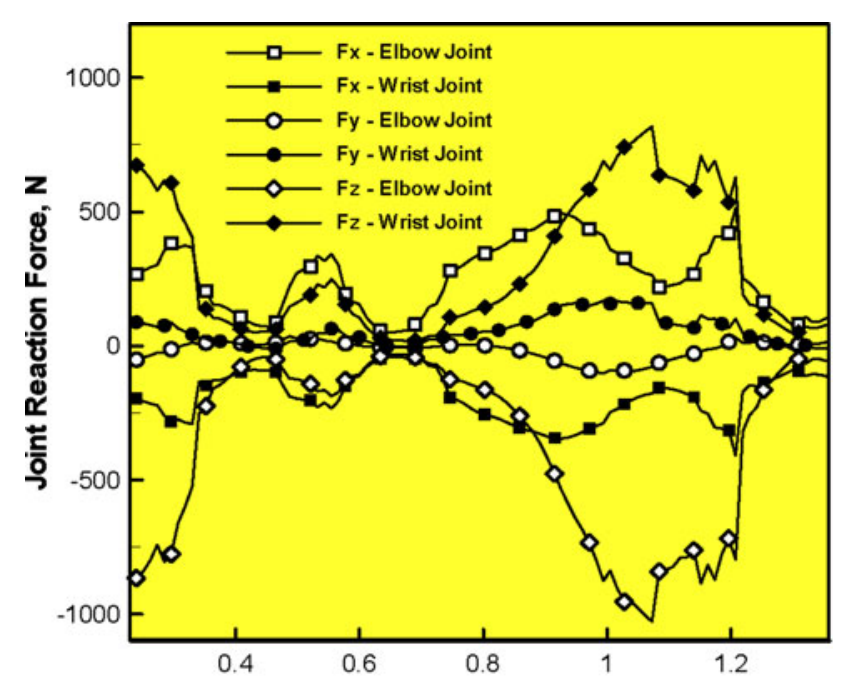

(a)

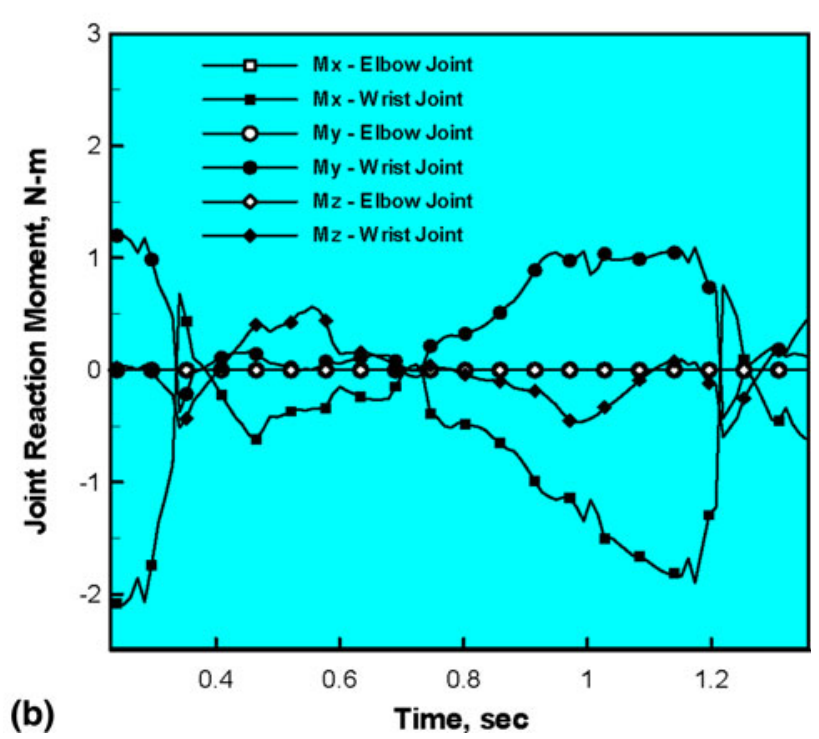

Fig. 9 Temporal evolution of the elbow and wrist joint reaction forces and moments over a single hand-push cycle

The latter findings are what makes the loading "non-proportional" and the fatigue-life prediction more complex. In other words, the stress state in general, and the maximum principal stress specifically do not scale linearly with the overall load magnitudes. Consequently, to reveal the stress state history of the fixation-plate during a single hand-push cycle, finiteelement analyses of the fractured radius subjected to the timevarying muscle forces and joint reaction-forces and moments had to be carried out multiple times (100 times in the present case) over a single hand-push cycle period.

3.2.4 High-Cycle Stress-Based Fatigue Analysis. Due to a relatively simple geometry of the fractured-radius fixation-plate implant and the fact that a pre-defined highcycle fatigue life is mandated for this component, it was deemed reasonable to assume that the fatigue-life of this component will be stress controlled. Furthermore it is assumed that the stress-based function responsible for the fatigue-induced failure is the maximum principal (tensile) stress. Next, stress-amplitude dependence of the number of cycles till failure is assumed to be defined by the traditional

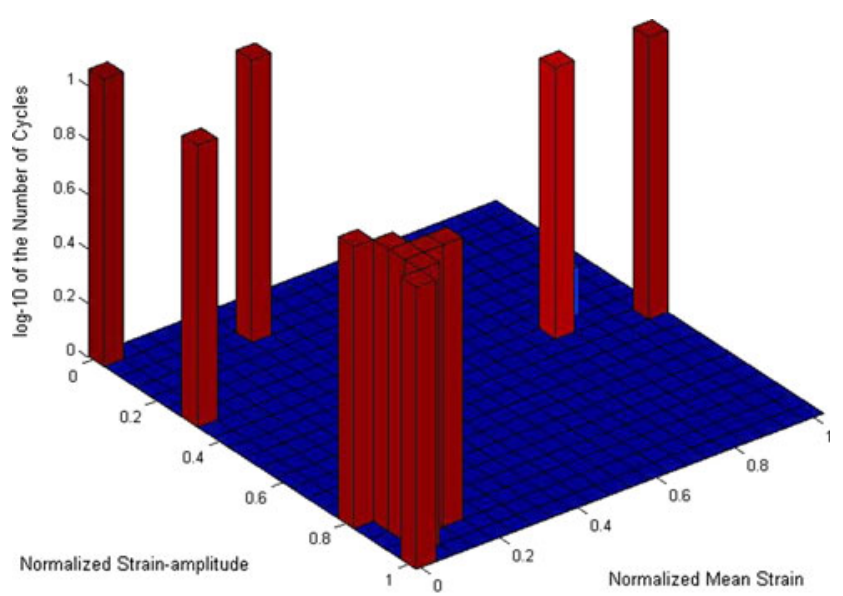

(a)

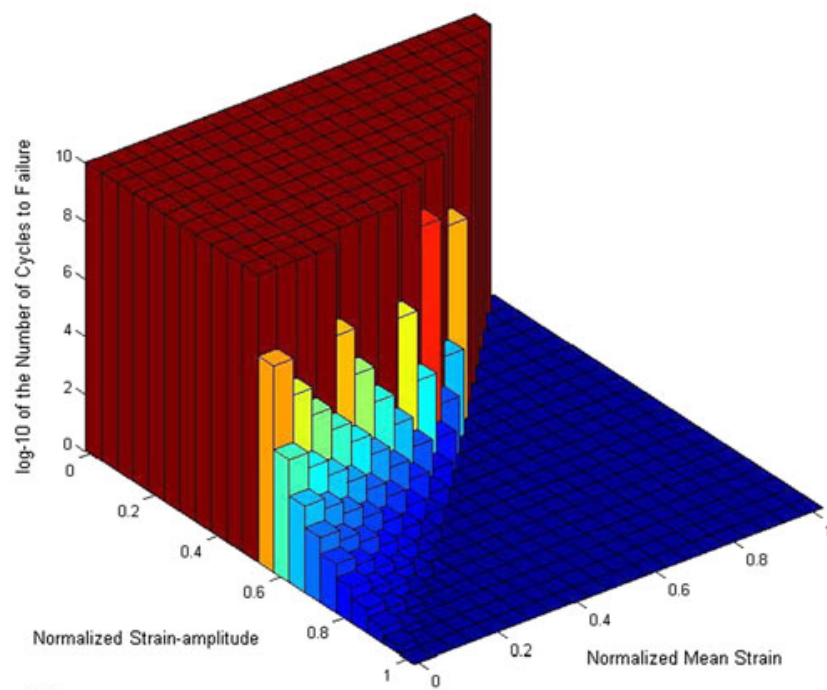

(b)

Fig. 10 Typical: (a) Rainflow cycle-counting analysis; and (b) Goodman-diagram procedure results obtained in the fixation-plate implant fatigue-life assessment procedure

Basquin relation (e.g., Ref 27) and the effect of the mean value of the maximum principal (tensile) stress is accounted for through the use of Goodman relation (e.g., Ref 27). The high-cycle fatigue parameters for Ti-6Al-4V are obtained from the Ansys fatigue material database (Ref 28).

To compute the number of cycles till failure for the given design/thickness of the fractured-radius fixation-plate implant, the procedure developed in our previous work was utilized (Ref 29). Due to space limitations, only a brief overview of this procedure will be provided here. The main steps of this procedure applied to each finite element of the fixation-plate implant include:

(a) utilization of the finite-element calculation results to determine temporal evolution of the maximum principal (tensile) stress/strain;

(b) application of the rainflow cycle counting analysis to determine a three-dimensional histogram relating the number of cycles with the maximum principal stress/strain amplitude and the associated mean value. 
An example of the typical results obtained in this portion of the work is displayed in Fig. 10(a);

(c) the use of the Goodman diagram for the calculation of the fractional damage associated with each load-cycle type (as characterized by a fixed value of the stress/ strain amplitude and the stress/strain mean value). An example of the typical results obtained in this portion of the work is displayed in Fig. 10(b); and

(d) computation of the total fractional damage associated with all load-cycle types and the use of the Miner's rule to compute the corresponding number of cycles till failure as an inverse of this fractional damage.

The fractured-radius fixation-plate implant life cycle is then set to that of its element associated with the smallest number of cycle till failure.

\section{Results and Discussion}

\subsection{Musculoskeletal Wheel-Chair Propulsion Analysis}

As mentioned earlier the sole purpose of conducting the musculoskeletal wheel-chair propulsion analysis was to obtain physiologically realistic loading conditions for the radius/ fixation-plate/screws assembly. Specifically, at each of
100 time increments during a single hand-push cycle, the muscle forces and the joint reaction forces and moments as well as the spatial position of the muscle attachment/via points and the joint-reaction points had to be obtained from the musculoskeletal analysis. In addition, the spatial position and the orientation of the radius/plate/screws assembly at each time increment had to be obtained from the musculoskeletal analysis.

An example of the temporal evolution of the forces and moments acting on the radius (at the elbow and wrist joint points) was shown earlier, Fig. 9(a) and (b). Similar results were obtained at muscle attachment/via points. As pointed out earlier these forces and moments are of non-constant amplitude and not in-phase resulting in non-proportional type of loading on the radius.

An example of the human-body/wheel-chair kinematics/ muscle-activity results at four time intervals during a single hand-push cycle is shown in Fig. 11(a)-(d). It should be noted that the activity of each muscle (i.e., the force produced by the muscle) is displayed pictorially in these figures by the thickness and the color shading of the line segments representing the muscles. Hence, the results displayed in Fig. 11(a)-(d) can be used to qualitatively assess how the activity/recruitment of different muscles is changing during a single hand-push cycle, e.g., variation in the activity of the Brachialis muscle is marked in these figures.
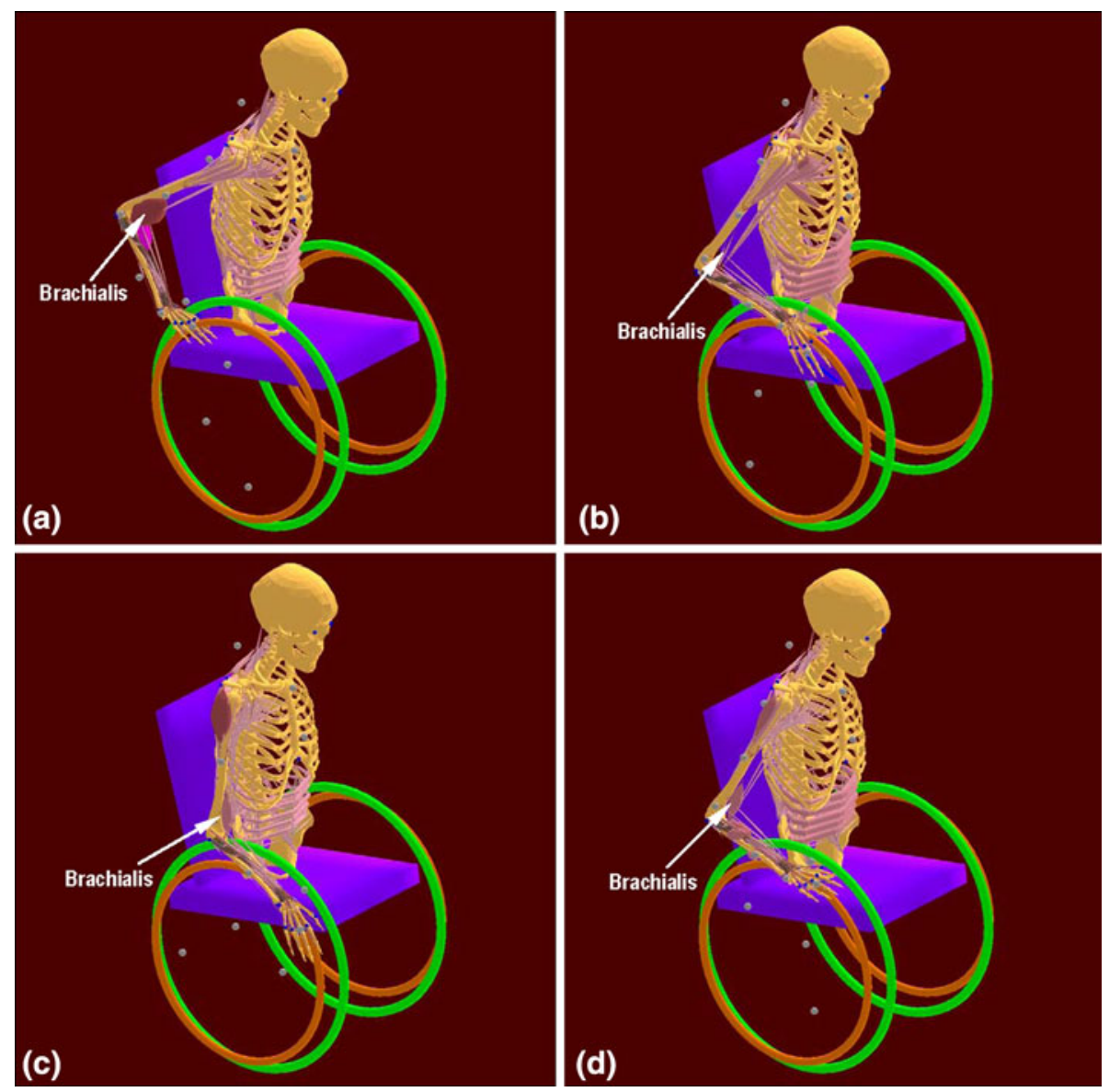

Fig. 11 Temporal evolution of the human-body kinematics and muscles activity at four equally spaced times during a single hand-push cycle 

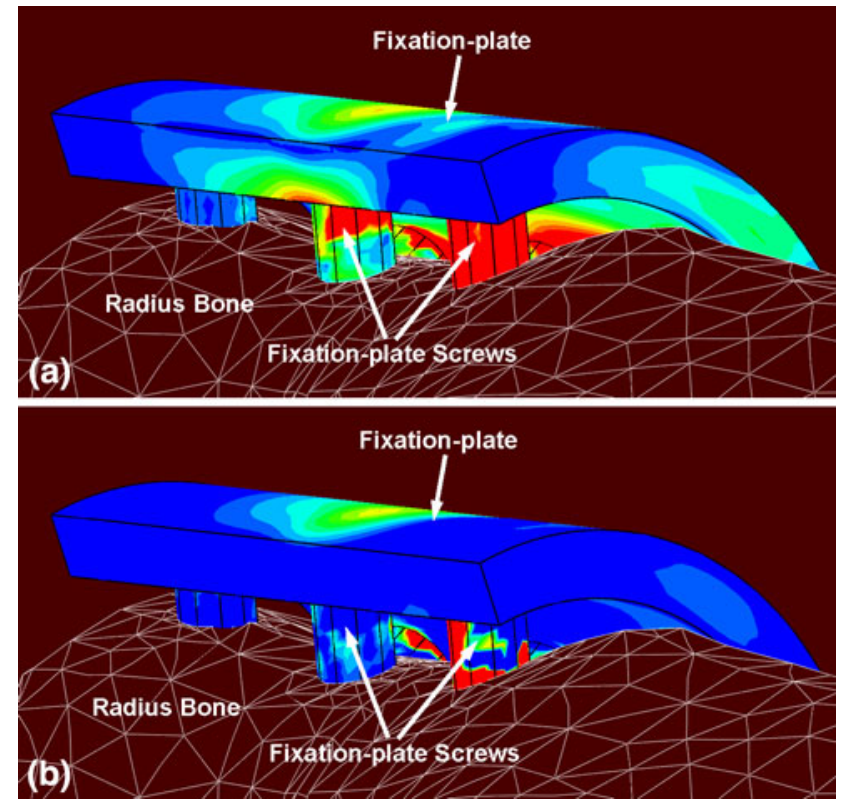

Fig. 12 Typical results pertaining to the spatial distribution of: (a) von-Mises equivalent stress $($ red $=30 \mathrm{MPa}$ and blue $=0.1 \mathrm{MPa}$ ); and (b) maximum principal stress (red $=20 \mathrm{MPa}$ and blue $=0.3 \mathrm{MPa}$ )

\subsection{Finite Element Results}

Since it was assumed throughout this work that the locking screws can secure well the fixation-plate implant to the two fractured-radius segments, the focus of the finite-element investigation was placed on the fixation-plate implant. The three primary functional requirements imposed onto the implant were: (a) sufficiently high strength to prevent any plastic deformation within the fixation plate; (b) adequate bending stiffness to prevent the two radius segments from intruding into each other; and (c) a pre-selected fatigue life expressed as a number of hand-push cycles. Using an expected implant resident time of 3 years and an average daily number of hand-push cycling of 1,000 , the implant fatigue life was set to two million cycles.

An example of the typical finite-element results is displayed in Fig. 12(a) and (b). The results displayed in Fig. 12(a) show that the von-Mises equivalent stress is substantially lower than the Ti-6Al-4V yield strength $(930 \mathrm{MPa})$. Thus, under all the loading and fixation-plate design conditions the implant strength requirement was found to be met. Likewise, the condition regarding the maximum interpenetration of the fracture surfaces of the radius was found to be satisfied (i.e., $<0.001 \mathrm{~m}$ ) for all combinations of loading conditions and the fixation-plate thickness.

As far as the fixation-plate implant fatigue-life requirements is concerned, it is found to be satisfied at some of the limits and not to be satisfied at the other limits of the implant-design/ positioning optimization variables. As mentioned earlier the fatigue-life in the present case is controlled by the maximum principal stress. Figure 12(b) displays an example of the typical results pertaining to spatial distribution of this stress component. Clearly the elements surrounding some of the screw-holes are associated with the highest levels of the maximum principal stress and are likely to control the overall fatigue-life of the component.

\subsection{Implant-Design/Optimization Results}

As stated earlier, under all the implant-design and muscle/ joint-imposed loading conditions, the functional requirements for the implant pertaining to its strength and bending stiffness were found to be satisfied. This is documented for the strength case in Fig. 13(a)-(c) in which the results of the DOE study are displayed for the von Mises stress as a function of the implant angular size and angular position at three different levels of the fixation-plate implant thickness. It is evident that the maximum von Mises stress for all the designs and all loading conditions represents only a minor fraction of the implant-material yield strength $(930 \mathrm{MPa})$. Based on these findings and the fact that the implant-design/positioning optimization is carried out within the same design domain used in the DOE study, the stiffness and strength requirements were not considered any further. The focus will be placed in this section on the functional requirement pertaining to the fatigue-life requirement of the implant.

Typical results pertaining to the progress of the implant design/positioning optimization are displayed in Fig. 14(a) and (b). In Fig. 14(a), the implant mass (objective function which is being minimized) is monitored as a function of the optimization iteration number. In Fig. 14(b), on the other hand, the corresponding implant fatigue-life (one of the constraining function, the minimum acceptable value which is set to two million cycles) is also monitored as a function of the optimization iteration number. The results displayed in Fig. 14(a) and (b) show that the optimized mass of the fractured-radius fixationplate implant is around $6.07 \mathrm{~g}$. This optimal implant design is associated with the following values of the three design/ positioning variables: (a) implant thickness of $3.06 \mathrm{~mm}$; (b) implant angular size of $41.09^{\circ}$; and (c) implant angular position of $105.7^{\circ}$.

\subsection{Material Selection}

Until this point in the present investigation, the same implant material, Ti-6Al-4V STA (Solution Treated and Aged) alloy was used. This is a commonly used fractured-radius fixation-plate implant material which provides a good combination of bio-compatibility, mechanical performance, and a low material/manufacturing cost. The present investigation has established that the key performance aspect of the fixationplate implant under consideration is fatigue life. While retaining the requirements concerning low material/manufacturing cost and bio-compatibility (ensured by carrying out material selection within the family of Ti-based alloys), a material selection procedure was conducted in the present work in order to identify potential material substitutes for Ti-6Al-4V.

While examining different Ti-based material alternatives, it was found that in all cases considered, strength requirement can be readily attained. Consequently, implant-material selection was carried out with respect to simultaneously, satisfying the implant stiffness and longevity requirements only and, at the end of the material-selection process, it was confirmed that the strength requirements were indeed satisfied. In all the cases considered, it was found that the longevity requirement is more difficult to meet. Consequently, in defining a single material selection parameter, a higher weighting factor $\left(w_{\mathrm{EL}}=0.8\right)$ was selected for endurance limit and a lower weighting factor $\left(w_{\mathrm{YM}}=0.2\right)$ for the Young's modulus of the candidate material. For convenience, the endurance limit and the Young's modulus 


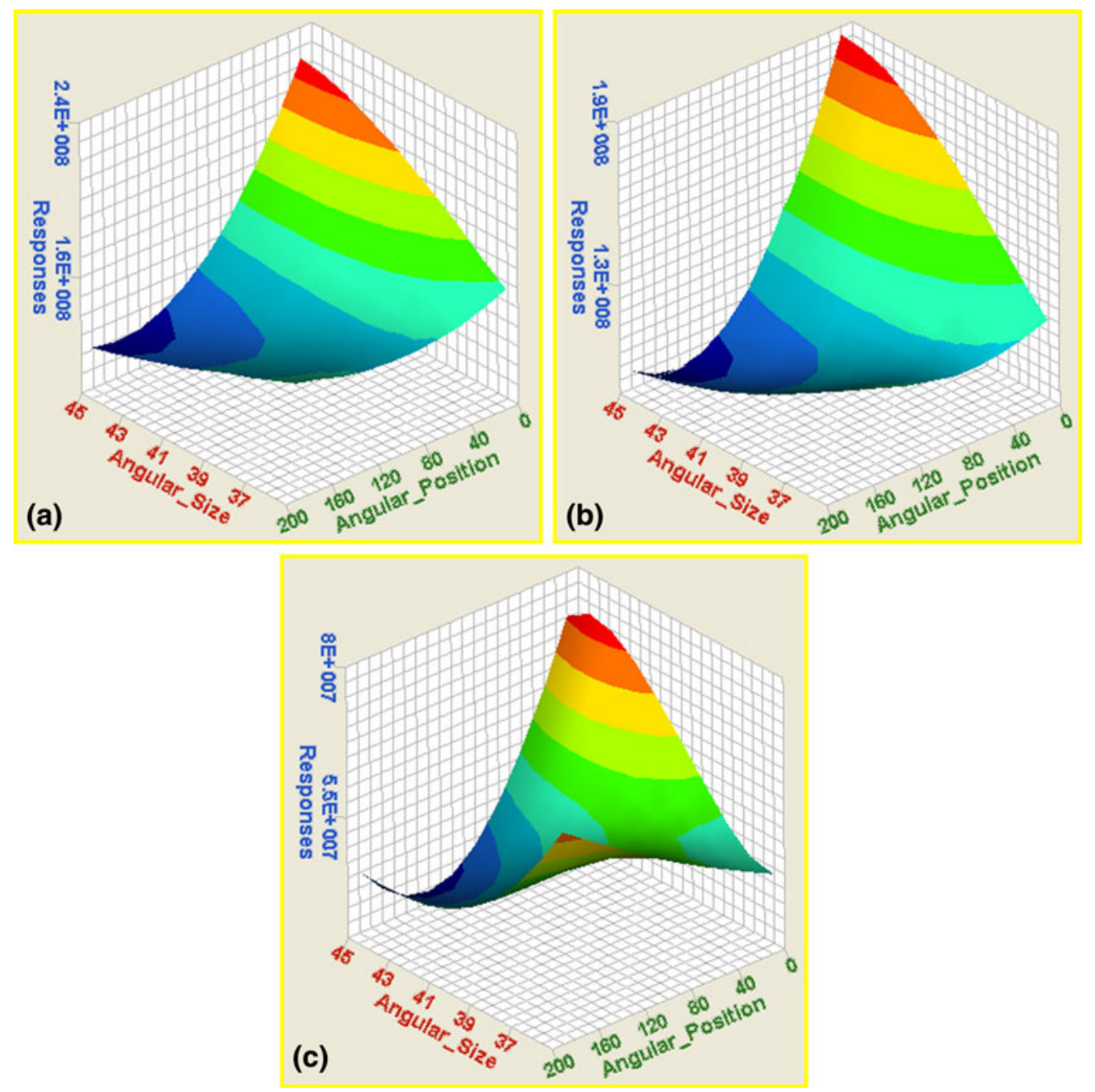

Fig. 13 Typical results of the Design of Experiments study pertaining to the variation of the maximum von Mises stress as a function of the fixation-plate angular size and angular position at three levels of the plate thickness: (a) $2 \mathrm{~mm}$; (b) $3 \mathrm{~mm}$; and (c) $4 \mathrm{~mm}$

of each candidate material are normalized by their respective counterparts in Ti-6Al-4V. Thus, the material selection index for the fixation-plate implant is defined as:

$M=w_{\mathrm{EL}} \frac{\sigma_{\mathrm{EL}}}{\sigma_{\mathrm{EL}, \mathrm{Ti}-6 \mathrm{Al}-4 \mathrm{~V}}}+w_{\mathrm{YM}} \frac{E}{E_{\mathrm{Ti}-6 \mathrm{Al}-4 \mathrm{~V}}}$

where $\sigma_{\mathrm{EL}}$ denotes endurance limit while $E$ is the Young's modulus. Clearly, $M=1.0$ for Ti-6Al-4V and for a material to be considered as a potential substitute for Ti-6Al-4V, its $M$ must be larger than 1.0.

It should be noted that the endurance limit of a material is typically obtained under zero mean-stress/strain loading conditions. Consequently, the use of this parameter in a material selection process does not take into account the important effect that mean stress/strain can have on the "infinite-life" material strength (i.e., the endurance limit). To overcome this problem, and still keep the material selection procedure relatively simple and manageable, a variable-mean stress/strain effective endurance limit is defined and used in Eq (5). This quantity is defined as a weighted average of the endurance-limit values associated with different mean-stress/strain levels encountered during a simple hand-push cycle. The weighting factors in these calculations were set to the corresponding number fractions of the given mean-stress/strain level encountered during a simple hand-push cycle.
To assist the implant material-selection process, a normalized stiffness $\left(E / E_{\mathrm{Ti}-6 \mathrm{Al}-4 \mathrm{~V}}\right)$ versus normalized endurance limit $\left(\sigma / \sigma_{\text {Ti-6Al-4V }}\right)$ plot is constructed in Fig. 15 . Few iso- $M$ lines are also drawn in this figure. The results displayed in Fig. 15 show that, with respect to the implant performance (as defined by its strength, stiffness, and longevity), Ti-10V-2Fe-3Al STA, Ti-3Al-8V-6Cr-4Zr-4Mo (Beta C), or Ti-15V-3Cr-3Sn-3Al STA may be a better alternative for the implant than Ti-6Al$4 \mathrm{~V}$. However, the final decision regarding the substitution of Ti-6Al-4V with these material alternatives should also account for material/manufacturing cost. Due to a lack of reliable/stable data pertaining to the cost of the materials in question, the effect of cost on material selection could not be taken into account in the present work.

\section{Summary and Conclusions}

Based on the work conducted and the results obtained in the present investigation, the following main summary remarks and conclusions can be drawn:

1. Design/positioning optimization of a fractured-radius fixation-plate implant is investigated computationally. 


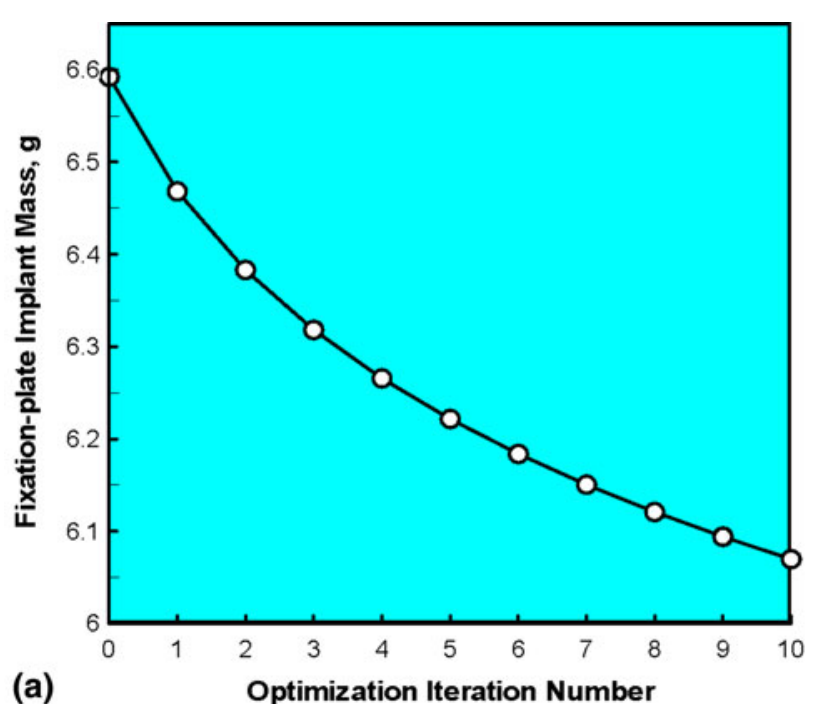

(a)

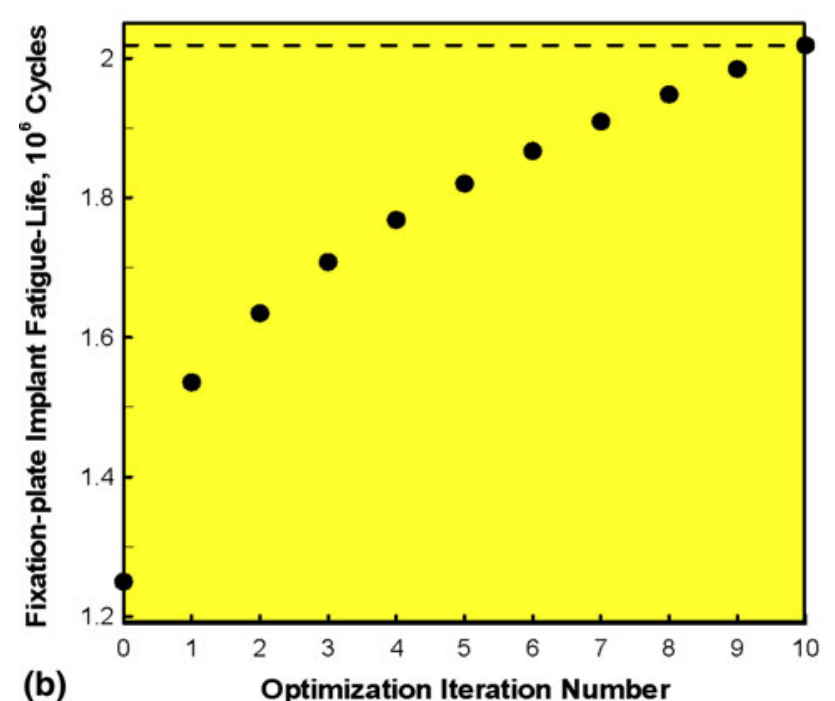

Fig. 14 Variations of: (a) the radius fixation-plate implant mass; and (b) radius implant fatigue-life, with the successive designoptimization iteration numbers

To provide the realistic physiological loading conditions experienced by the implant during normal daily activities of the patient, a musculoskeletal multi-body dynamics analysis is coupled with implant finite-element/designoptimization methods.

2. The results show that out of the three functional requirements placed on the implant (i.e., its strength, bending stiffness, and longevity), it is longevity which typically controls the implant optimal design/positioning.

3. Under an assumption that the musculoskeletal multi-body dynamics analysis of the wheel-chair propulsion can be used to mimic the most critical loading conditions experienced by the radius-fixation implant during normal daily activities of the human with a surgically implanted radius-fixation plate, the optimal implant design and positioning were determined with respect to the attainment of a minimal implant mass.

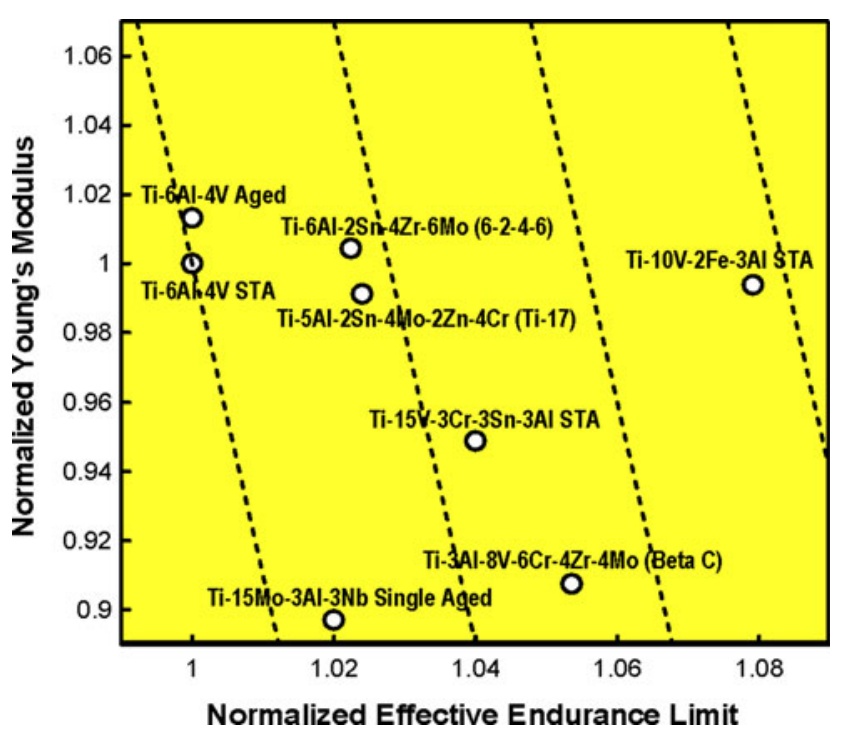

Fig. 15 Material selection chart used in the analysis of potential replacement of Ti-6Al-4V as the fixation-plate implant material with other Ti-based alloys

4. In the final portion of the article, potential material replacements have been considered for Ti-6Al-4V, the alloy used in the present finite-element/design-optimization analysis of a radius-fixation implant, in order to further reduce implant thickness.

\section{Acknowledgments}

A portion of the material presented in this article is based on the use of the AnyBody Modeling System, a musculoskeletal multibody dynamics software (Ref 6). The authors are indebted to Ozen Engineering for donating an AnyBody Modeling System license to Clemson University. One of the authors (G. A.) would like to acknowledge the support of Altair Engineering under the Altair University Fellowship program.

\section{References}

1. K.C. Kim, J.K. Lee, D.S. Hwang, J.Y. Yang, and Y.M. Kim, Distal Hybrid Interlocking in the Femoral Shaft Fracture, Orthopedics, 2007, 30, p 605

2. M. Sivasankar, S.K. Dwivedy, and D. Chakraborty, Fatigue Analysis of Artificial Hip Joints for Different Activities, 2nd International Congress on Computational Mechanics and Simulation, Guwahati, India, 2006

3. D.D. D'Lima, N. Steklov, B.J. Fregly, S.A. Banks, and C.W. Colwell, Jr., In Vivo Contact Stresses during Activities of Daily Living after Knee Arthroplasty, J. Orthop. Res., 2008, 26, p 1549-1555

4. D.O. O'Connor, D.W. Burke, M. Jasty, R.C. Sedlacek, and W.H. Harris, In Vitro Measurement of Strain in the Bone Cement Surrounding the Femoral Component of Total Hip Replacements During Simulated Gait and Stair-Climbing, J. Orthop. Res., 1996, 14(5), p 769-777

5. L. Cristofolini, P. Savigni, A.S. Teutonico, and M. Viceconti, In Vitro Load History to Evaluate the Effects of Daily Activities on Cemented Hip Implants, Acta Bioeng. Biomech., 2003, 5(2), p 74-88

6. AnyBody 3.0, AnyBody Technology A/S, Aalborg, Denmark, 2008 
7. M. Damsgaard, J. Rasmussen, S.T. Christensen, E. Surma, and M. de Zee, Analysis of Musculoskeletal Systems in the AnyBody Modeling System, Simul. Model. Pract. Theory, 2006, 14, p 1100-1111

8. J. Rasmussen and M. de Zee, Design Optimization of Airline Seats, SAE Conference, SAE No. 2008-01-1863, 2008

9. S. Dendorfer and S. Torholm, Final Report on Feasibility Study, Report No: 21385/08/NL/PA, Presented to ESTEC/ESA by AnyBody Technology $A / S$, May, 2008

10. AnyScript Model Repository 7.1, AnyBody 3.0, AnyBody Technology A/S, Aalborg, Denmark, 2009

11. F.C.T. Van der Helm, A Finite Element Musculoskeletal Model of the Human Shoulder Mechanism, J. Biomech., 1994, 27, p 551-569

12. M. de Zee, L. Hansen, C. Wong, J. Rasmussen, and E.B. Simonsen, A Generic Detailed Rigid-body Lumbar Spine Model, J. Biomech., 2007, 40, p 1219-1227

13. H. Wilke, P. Neef, B. Hinz, H. Seidel, and L. Claes, Intradiscal Pressure Together with Anthropometric Data-A Data Set for the Validation of Models, Clin. Biomech., 2001, 16(Suppl. 1), p S111-S126

14. P. de Jong, M. de Zee, P.A.J. Hilbers, H.H.C.M. Savelberg, F.N. van de Vosse, A. Wagemakers, and K. Meijer, "Multi-body Modeling of Recumbent Cycling: An Optimization of Configuration and Cadence," Master's Thesis Medical Engineering, TU/e Biomodelling and Bioinformatics, University of Maastricht, Movement Sciences, Aalborg University, 2006

15. K.N. An, B.M. Kwak, E.Y. Chao, and B.F. Morrey, Determination of Muscle and Joint Forces: A New Technique to Solve the Indeterminate Problem, J. Biomech. Eng., 1984, 106, p 364-367

16. Rancho Los Amigos National Rehabilitation Center, http://www. rancho.org/research home.htm

17. J. Rasmussen, S. Torholm, and M. de Zee, Computational Analysis of the Influence of Seat Pan Inclination and Friction on Muscle
Activity and Spinal Joint Forces, Int. J. Industr. Ergon., 2009, 39, p 52-57

18. Z. Yosibash, R. Padan, L. Joscowicz, and C. Milgrom, A CT-based High-order Finite Element Analysis of the Human Proximal Femur Compared to In Vitro Experiments, ASME J. Biomech. Eng., 2007, 129(3), p 297-309

19. Mimics, Medical Imaging Software, Materialise, User Documentation, 2009

20. ABAQUS Version 6.8-1, User Documentation, Dassault Systems, 2009

21. P.Y. Papalambros and D.J. Wilde, Principles of Optimal Design: Modeling and Computation, 2nd ed., Cambridge University Press, Cambridge, UK, 2000

22. M.P. Bendsoe and N. Kikuchi, Generating Optimal Topologies in Structural Design Using a Homogenization Method, Comput. Methods Appl. Mech. Eng., 1988, 71(2), p 197-224

23. R.T. Haftka and R.V. Grandhi, Structural Shape Optimization: A Survey, Comput. Methods Appl. Mech. Eng., 1986, 57(1), p 91106

24. HyperStudy, User Manual, Altair Engineering Inc., Troy, MI, 2009

25. U. Schramm, Multi-Disciplinary Optimization for NVH and Crashworthiness, Altair Engineering Inc., Troy, MI, 2007

26. M. Matsuishi and T. Endo, Fatigue of Metals Subjected to Varying Stress, Proceedings of Kyushi Branch, 1987

27. P. Brondsted, H. Lilholt, and A. Lystrup, Composite Materials for Wind Power Turbine Blades, Annu. Rev. Mater. Res., 2005, 35, p 505-538

28. ANSYS Version 12, User Documentation, Ansys Inc., 2009

29. M. Grujicic, G. Arakere, W.C. Bell, H. Marvi, H.V. Yalavarthy, B. Pandurangan, I. Haque, and G.M. Fadel, Reliability-based Design Optimization for Durability of Ground-vehicle Suspension-system Components, J. Mater. Eng. Perform., Accepted for Publication, April 2009 